\title{
Classification of two-qubit states
}

\author{
Paweł Caban ${ }^{1}$ - Jakub Rembieliński ${ }^{1}$. \\ Kordian A. Smoliński ${ }^{1}$ - Zbigniew Walczak ${ }^{1}$
}

Received: 16 June 2015 / Accepted: 3 September 2015 / Published online: 18 September 2015 (C) The Author(s) 2015. This article is published with open access at Springerlink.com

\begin{abstract}
Verstraete, Dehaene and DeMoor showed that each of the two-qubit states can be generated from one of two canonical families of two-qubit states by means of transformations preserving the tensor structure of the state space. Precisely, each of such states can be generated from a three-parameter family of Bell-diagonal states or from three-parameter rank-deficient states. In this paper, we show that this classification of two-qubit states can be refined. In particular, we show that the latter canonical family of states can be reduced to three fixed states and a two-parameter family of two-qubit states. For this family of states, we provide a simple parametrization that guarantees positive semidefiniteness of the states and enables easier calculation of the Wootters concurrence and quantum discord. Moreover, we present a new general parametrization of all two-qubit states generated from the canonical families of states using sets of (pseudo)orthogonal four-vectors (frames). An advantage of the presented approach lies in the fact that the standard conditions for positive semidefiniteness of states are equivalent to (pseudo)orthogonality conditions for four-vectors serving as
\end{abstract}

This work has been supported by the Polish National Science Centre under the contract 2014/15/B/ST2/00117 and by the University of Lodz.

$凶 \quad$ Paweł Caban

P.Caban@merlin.phys.uni.lodz.pl

Jakub Rembieliński

jaremb@uni.lodz.pl

Kordian A. Smoliński

K.A.Smolinski@merlin.phys.uni.lodz.pl

Zbigniew Walczak

Z.Walczak@merlin.phys.uni.lodz.pl

1 Department of Theoretical Physics, University of Lodz, Pomorska 149/153, 90-236 Lodz, Poland 
parameters (and appropriate conditions for parameters of the corresponding canonical family of states).

Keywords Quantum computing - Quantum mechanics · Two-qubit state · Quantum discord

\section{Introduction}

The vast majority of quantum information protocols exploit quantum correlations. Various notions of quantumness of correlations exist in the literature (see, for example, $[1,2])$. We restrict our attention to the simplest non-trivial case of two-partite systemtwo-qubit system. The space of states of such a system is of the form $\mathscr{H}=\mathscr{H}_{A} \otimes \mathscr{H}_{B}$, where $\mathscr{H}_{A}=\mathbb{C}^{2}$ and $\mathscr{H}_{B}=\mathbb{C}^{2}$ are spaces of states of systems $\mathrm{A}$ and $\mathrm{B}$, respectively. Therefore, the most general transformations considered in the context of quantum correlations have to preserve the tensor structure of the space $\mathscr{H}$. Such transformations are of the form

$$
\rho \mapsto(A \otimes B) \rho(A \otimes B)^{\dagger},
$$

where $A, B$ are $2 \times 2$ matrices.

The main goal of our paper is to discuss the classification of all two-qubit states with respect to transformations preserving the tensor product structure of the state space. This last condition is important from the physical point of view since each partition of a quantum system into subsystems corresponds to a decomposition of the space of states into the tensor product of subsystem spaces. Also the entanglement of a physical system is always relative to the tensor product decomposition [3]. As a consequence, the following question arises: what is the structure of the two-qubit space of states if we take into account transformations (1).

In [4], it was shown that each of two-qubit states can be generated from one of the two canonical families of two-qubit states by means of transformations (1) (provided that det $A \neq 0$, det $B \neq 0$ ). Precisely, each of such states can be generated from a threeparameter family of Bell-diagonal states or from three-parameter rank-deficient states (in [4] rank-deficient states are described by four parameters but one parameter can be eliminated by normalization). However, as we show below, the classification given in [4] can be refined. Since the Bell-diagonal states are widely discussed in the literature, we focus our attention on the latter family of states. In particular, we will show that this three-parameter family of states should be replaced by three fixed states and a twoparameter family of states. We show also that this set of states is a minimal generating set with respect to transformations (1). It is worth to note that, in contrast to the Belldiagonal states, in these cases it is impossible to depolarize both subsystem states by means of transformations (1). We find an appropriate parametrization guaranteeing positive semidefiniteness of states from the two-parameter family. Moreover, for those states we give explicit (and compact) formulas for quantum discord.

Furthermore, for all canonical families of states we determine transformations leaving them invariant (stability groups). This can be useful in further investigations of the structure of state space. We give also a new general parametrization of all twoqubit states generated from the corresponding canonical families. This parametrization 
employs (pseudo)orthogonal frames. For a general two-qubit state parametrized in this way, the positive semidefiniteness conditions for the density matrix are equivalent to (pseudo)orthogonality conditions for four-vectors serving as parameters (and appropriate conditions for parameters of the corresponding canonical family of states).

The proof of our classification theorem is based on the canonical homomorphism of the SL $(2, \mathbb{C})$ group onto the proper orthochronous Lorentz group. We utilize also the theory of vector spaces in which the usual positive definite inner product is replaced by an indefinite one. Thus, for the readers convenience, we have collected basic results in "Appendix 1."

\section{Two-qubit states}

We consider here two-qubit states, i.e., states acting in the Hilbert space $\mathscr{H}=\mathscr{H}_{A} \otimes$ $\mathscr{H}_{B}$, where $\mathscr{H}_{A}=\mathscr{H}_{B}=\mathbb{C}^{2}$. An arbitrary $4 \times 4$ matrix can be written in the following form:

$$
\rho=\frac{1}{4} \sum_{\mu, \nu=0}^{3} R^{\mu \nu} \sigma_{\mu} \otimes \sigma_{\nu},
$$

where $\sigma_{0}=I, \sigma_{i}, i=1,2,3$, are Pauli matrices. A matrix $\rho$ corresponds to a quantum state iff it is hermitian, positive semidefinite and has a unit trace. The hermiticity of $\rho$ is equivalent to the condition that the matrix $R=\left[R^{\mu \nu}\right]$ is real. The unit trace of $\rho$ can be obtained by a proper normalization. However, checking if a matrix $\rho$ is positive semidefinite requires more effort. For example, one can use the following property [5]: A hermitian matrix $\rho$ is positive semidefinite iff all of the coefficients

$$
\begin{aligned}
& s_{1}=\operatorname{Tr} \rho, \\
& s_{2}=\frac{1}{2}\left(s_{1} \operatorname{Tr} \rho-\operatorname{Tr} \rho^{2}\right), \\
& s_{3}=\frac{1}{3}\left(s_{2} \operatorname{Tr} \rho-s_{1} \operatorname{Tr} \rho^{2}+\operatorname{Tr} \rho^{3}\right), \\
& s_{4}=\frac{1}{4}\left(s_{3} \operatorname{Tr} \rho-s_{2} \operatorname{Tr} \rho^{2}+s_{1} \operatorname{Tr} \rho^{3}-\operatorname{Tr} \rho^{4}\right),
\end{aligned}
$$

are nonnegative.

Notice that local transformations

$$
\rho \mapsto(A \otimes B) \rho(A \otimes B)^{\dagger}, \quad A, B \in \operatorname{SL}(2, \mathbb{C}),
$$

are completely positive maps, as they are directly written in the so-called Kraus form and therefore preserve positive semidefiniteness of matrix $\rho$, and moreover, they preserve the tensor product structure of the space $\mathscr{H}=\mathscr{H}_{A} \otimes \mathscr{H}_{B}$.

Now, it is well known that there exists a canonical homomorphism from $\operatorname{SL}(2, \mathbb{C})$ group into Lorentz group. In fact, in this homomorphism we can obtain only proper orthochronous Lorentz transformations, that is it is a homomorphism from $\operatorname{SL}(2, \mathbb{C})$ onto $L_{+}^{\uparrow}$. Let $\Lambda_{1}$ and $\Lambda_{2}$ be images of $A$ and $B$ in this homomorphism, respectively. One can easily see that the transformation (4) corresponds to the following transformation of a matrix $R$ defined in Eq. (2): 


$$
R \mapsto \Lambda_{1} R \Lambda_{2}^{\mathrm{T}}
$$

Our main result is summarized in the following theorem:

Theorem 1 (Canonical form of two-qubit state) A two-qubit density matrix $\rho$ written in the form (2) can be generated via the transformation

$$
\rho=\frac{(A \otimes B) \tilde{\rho}(A \otimes B)^{\dagger}}{\operatorname{Tr}\left[(A \otimes B) \tilde{\rho}(A \otimes B)^{\dagger}\right]}, \quad A \otimes B \in S L(2, \mathbb{C}) \otimes S L(2, \mathbb{C})
$$

from a density matrix $\tilde{\rho}$, where

$$
\tilde{\rho}=\frac{1}{4} \sum_{\mu, \nu=0}^{3} \Sigma^{\mu \nu} \sigma_{\mu} \otimes \sigma_{\nu}
$$

and $\Sigma$ has one of the following forms:

Case I:

$$
\Sigma_{l}=\left(\begin{array}{llll}
1 & 1 & 0 & 0 \\
0 & 0 & 0 & 0 \\
0 & 0 & 0 & 0 \\
0 & 0 & 0 & 0
\end{array}\right), \quad \text { or } \quad \Sigma_{l}^{\prime}=\left(\begin{array}{llll}
1 & 0 & 0 & 0 \\
1 & 0 & 0 & 0 \\
0 & 0 & 0 & 0 \\
0 & 0 & 0 & 0
\end{array}\right)
$$

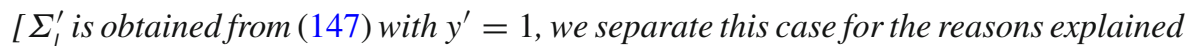
in "Appendix 2"],

Case II:

$$
\Sigma_{\|}=\left(\begin{array}{llll}
1 & 1 & 0 & 0 \\
1 & 1 & 0 & 0 \\
0 & 0 & 0 & 0 \\
0 & 0 & 0 & 0
\end{array}\right)
$$

Case III:

$$
\Sigma_{I I I}=\left(\begin{array}{llll}
1 & 0 & 0 & 0 \\
0 & X & 0 & 0 \\
0 & 0 & Y & 0 \\
0 & 0 & 0 & Z
\end{array}\right)
$$

where we can choose $1 \geq X \geq Y \geq|Z|$ and the following conditions hold

$$
\begin{aligned}
& X^{2}+Y^{2}+Z^{2} \leq 3, \\
& X^{2}+Y^{2}+Z^{2}+2 X Y Z \leq 1, \\
& (1-X-Y-Z)(1-X+Y+Z)(1+X-Y+Z)(1+X+Y-Z) \geq 0,
\end{aligned}
$$

Case IV:

$$
\Sigma_{I V}=\left(\begin{array}{cccc}
1 & 0 & 0 & y \\
0 & x & 0 & 0 \\
0 & 0 & x & 0 \\
y & 0 & 0 & 2 y-1
\end{array}\right)
$$


with $0 \leq x<1,0<y<1-x$.

The transformation (6) corresponds to the following transformation of $\Sigma$ :

$$
R=\frac{\Lambda_{1} \Sigma \Lambda_{2}^{T}}{\left(\Lambda_{1} \Sigma \Lambda_{2}^{T}\right)^{00}} .
$$

The detailed proof of Theorem 1 is presented in "Appendix 2."

Before further discussion of states $\Sigma_{1}, \Sigma_{\mathrm{I}}, \Sigma_{\mathrm{lII}}$, and $\Sigma_{\mathrm{lV}}$, let us recall some properties of the canonical homomorphism $\operatorname{SL}(2, \mathbb{C}) \rightarrow L_{+}^{\uparrow}$. For arbitrary four-vector $a$ we consider a $2 \times 2$ hermitian matrix $a \sigma=a^{\mu} \sigma_{\mu}=a^{0} I+\mathbf{a} \cdot \sigma$. It holds $\operatorname{det}(a \sigma)=$ $a^{0^{2}}-\mathbf{a}^{2}$. For each $A \in \operatorname{SL}(2, \mathbb{C})$ we define a transformation

$$
a \sigma \mapsto A(a \sigma) A^{\dagger}=a^{\prime} \sigma
$$

We see that $\operatorname{det}\left[A(a \sigma) A^{\dagger}\right]=\operatorname{det}(a \sigma)$, therefore $a^{\prime}=\Lambda(A) a$, where $\Lambda(A) \in L_{+}^{\uparrow}$. In this way, we have defined the canonical homomorphism $A \mapsto \Lambda(A)$. Notice that for arbitrary four-vectors $a$ and $b$, the scalar product

$$
a b=\eta_{\mu \nu} a^{\mu} b^{\nu}=\frac{1}{4}[\operatorname{det}(a \sigma+b \sigma)-\operatorname{det}(a \sigma-b \sigma)],
$$

where the Minkowski metric tensor $\left[\eta_{\mu \nu}\right]=\operatorname{diag}(1,-1,-1,-1)$ is preserved under the transformations (14).

Now, denoting

$$
\tilde{e}^{0}=\left(\begin{array}{l}
1 \\
0 \\
0 \\
0
\end{array}\right), \quad \tilde{e}^{1}=\left(\begin{array}{l}
0 \\
1 \\
0 \\
0
\end{array}\right), \quad \tilde{e}^{2}=\left(\begin{array}{l}
0 \\
0 \\
1 \\
0
\end{array}\right), \quad \tilde{e}^{3}=\left(\begin{array}{l}
0 \\
0 \\
0 \\
1
\end{array}\right),
$$

we have

$$
\sigma_{\alpha}=\left(\tilde{e}^{\alpha}\right)^{v} \sigma_{v}
$$

and

$$
\tilde{e}^{\alpha} \tilde{e}^{\beta}=\eta_{\mu \nu}\left(\tilde{e}^{\alpha}\right)^{\mu}\left(\tilde{e}^{\beta}\right)^{\nu}=\eta^{\alpha \beta} .
$$

Thus, the four-vectors $\left\{\tilde{e}^{\alpha}\right\}$ form a (pseudo)orthogonal frame. Notice that $\alpha, \beta, \ldots$ enumerate four-vectors in a frame, while $\mu, v, \ldots$ enumerate components of fourvectors. Furthermore, under the transformation (14)

$$
\tilde{e}^{\alpha} \sigma \mapsto e^{\alpha} \sigma=A\left(\tilde{e}^{\alpha} \sigma\right) A^{\dagger}, \quad e^{\alpha}=\Lambda(A) \tilde{e}^{\alpha}
$$

and transformed four-vectors $\left\{e^{\alpha}\right\}$ form a frame, too:

$$
e^{\alpha} e^{\beta}=\eta^{\alpha \beta}
$$

Notice the obvious fact that $\tilde{e}^{0}$ (and consequently $e^{0}$ ) is a time-like four-vector, while $\tilde{e}^{i}$ (and consequently $e^{i}$ ) are space-like four-vectors. 
Next, one-qubit state can be written as

$$
u \sigma=u^{\mu} \sigma_{\mu}=u^{0} \sigma_{0}+\mathbf{u} \cdot \sigma
$$

with $u^{0}=1 / 2, \mathbf{u}=\mathbf{n} / 2$ and $\mathbf{n}^{2} \leq 1$. Note that for pure states $\mathbf{n}^{2}=1$ and consequently the four-vector $u^{\mu}$ is light-like (with $u^{0}>0$ ), while for mixed states $u^{\mu}$ is time-like (with $u^{0}>0$, too). The space-like case of $u^{\mu}$ is excluded. Moreover, the transformation

$$
\frac{A(u \sigma) A^{\dagger}}{\operatorname{Tr}\left[A(u \sigma) A^{\dagger}\right]}=\frac{u^{\prime} \sigma}{\operatorname{Tr}\left(u^{\prime} \sigma\right)},
$$

where $A \in \operatorname{SL}(2, \mathbb{C}), u^{\prime}=\Lambda(A) u$ preserves the character of the state, i.e., if $u$ is light-like, then $u^{\prime}$ is light-like, too, and similarly for time-like $u$.

Now, subsystems in two-qubit state are one-qubit states obtained by partial traces. Therefore, their properties are the same as discussed above. In particular, for time-like and light-like four-vectors $u^{\mu}$, we have $\operatorname{Tr}(u \sigma) \neq 0$, and consequently, the transformations (6) are well defined.

Note that under the transformation (6), the Wootters concurrence [6] changes as [4]:

$$
C(\rho)=\frac{C(\tilde{\rho})}{\operatorname{Tr}\left[(A \otimes B) \tilde{\rho}(A \otimes B)^{\dagger}\right]} .
$$

Now, let us analyze the cases $\Sigma_{\mathrm{l}}, \Sigma_{\mathrm{II}}, \Sigma_{\mathrm{III}}$, and $\Sigma_{\mathrm{IV}}$ in detail.

\subsection{The orbit generated by the states $\Sigma_{1}$ and $\Sigma_{1}^{\prime}$ [Eq. (8)]}

The density matrix corresponding to $\Sigma_{1}$ has the following form:

$$
\tilde{\rho}_{1}=\frac{1}{4} \sigma_{0} \otimes\left(\sigma_{0}+\sigma_{1}\right) \text {. }
$$

This is a mixed, separable state, and after the transformation (6), it takes the form

$$
\tilde{\rho}_{1} \mapsto \rho_{1}=\frac{\left(e^{0} \sigma\right) \otimes\left(\left(f^{0}+f^{1}\right) \sigma\right)}{4\left(e^{0}\right)^{0}\left[\left(f^{0}\right)^{0}+\left(f^{1}\right)^{0}\right]},
$$

where four-vectors $\left\{e^{\alpha}\right\}$ are defined by (19) and fulfill the condition (20), and the same relations are fulfilled by four-vectors $\left\{f^{\alpha}\right\}$. Notice that $e^{0}$ is a time-like four-vector, while $f^{0}+f^{1}$ is a light-like four-vector.

Equation (25) gives the general parametrization of the orbit generated by the state $\Sigma_{1}$. Similarly, the density matrix corresponding to $\Sigma_{1}^{\prime}$ has the form

$$
\tilde{\rho}_{\mathrm{l}}^{\prime}=\frac{1}{4}\left(\sigma_{0}+\sigma_{1}\right) \otimes \sigma_{0}
$$

This is also a mixed, separable state, and the transformation (6) transforms it to

$$
\tilde{\rho}_{\text {। }}^{\prime} \mapsto \rho_{\text {। }}^{\prime}=\frac{\left(\left(e^{0}+e^{1}\right) \sigma\right) \otimes\left(f^{0} \sigma\right)}{4\left[\left(e^{0}\right)^{0}+\left(e^{1}\right)^{0}\right]\left(f^{0}\right)^{0}},
$$


where $e^{0}, e^{1}$, and $f^{0}$ have the same meaning as in (25). Here, $e^{0}+e^{1}$ is a lightlike four-vector, while $f^{0}$ is a time-like four-vector. Equation (27) gives the general parametrization of the orbit generated by the state $\Sigma_{1}^{\prime}$.

Notice that the states (25) and (27) have the form of the tensor product of a pure and mixed state.

\subsection{The orbit generated by the state $\Sigma_{\mathrm{II}}[\mathrm{Eq} \cdot(9)]$}

The corresponding density matrix has the following form:

$$
\tilde{\rho}_{\|}=\frac{1}{4}\left(\sigma_{0}+\sigma_{1}\right) \otimes\left(\sigma_{0}+\sigma_{1}\right) .
$$

This is a pure, separable state. Therefore, all states generated from $\tilde{\rho}_{\| \mid}$via the transformation (6)

$$
\tilde{\rho}_{\| \mid} \mapsto \rho_{\|}=\frac{(a \sigma) \otimes(b \sigma)}{4 a^{0} b^{0}},
$$

where $a=e^{0}+e^{1}$ and $b=f^{0}+f^{1}$ are light-like and are also pure and separable. This statement follows from Eq. (23) and from the observation that pure states are transformed into pure ones under (6). The opposite statement is also true: all pure, separable two-qubit states can be generated from $\tilde{\rho}_{\text {II }}$ via the transformation (6). Indeed, any pure, separable, two-qubit state can be written as

$$
\frac{1}{2}(I+\mathbf{n} \cdot \boldsymbol{\sigma}) \otimes \frac{1}{2}(I+\tilde{\mathbf{n}} \cdot \boldsymbol{\sigma}), \quad \mathbf{n}^{2}=\tilde{\mathbf{n}}^{2}=1 .
$$

The above state corresponds to $\rho_{\mathrm{ll}}$ given in (29) if we take $a=\left(\frac{1}{2}, \frac{\mathbf{n}}{2}\right)$ and $b=$ $\left(\frac{1}{2}, \frac{\tilde{\mathbf{n}}}{2}\right)$. Observe that such defined $a$ and $b$ are light-like. Now, arbitrary light-like fourvector can be generated via Lorentz transformation from the standard four-vector $(1,1,0,0)$. This proves our statement.

\subsection{The orbit generated by the state $\Sigma_{\mathrm{III}}[\mathrm{Eq} \cdot(10)]$}

The corresponding density matrix has the following form

$$
\tilde{\rho}_{\mathrm{III}}(X, Y, Z)=\frac{1}{4}\left(\begin{array}{cccc}
1+Z & 0 & 0 & X-Y \\
0 & 1-Z & X+Y & 0 \\
0 & X+Y & 1-Z & 0 \\
X-Y & 0 & 0 & 1+Z
\end{array}\right),
$$

with the conditions (11) and $1 \geq X \geq Y \geq|Z|$. The concurrence of this state is equal to

$$
C\left(\tilde{\rho}_{\mathrm{III}}(X, Y, Z)\right)= \begin{cases}0 & \text { if } \quad 1+X+Y+Z \geq 0 \\ -\frac{1}{2}(1+X+Y+Z) & \text { if } \quad 1+X+Y+Z<0\end{cases}
$$


All states generated from $\tilde{\rho}_{\mathrm{III}}$ via the transformation (6) can be written in the following form

$$
\begin{aligned}
\tilde{\rho}_{\mathrm{III}} \mapsto \rho_{\mathrm{III}}= & \frac{1}{4 N}\left[\left(e^{0} \sigma\right) \otimes\left(f^{0} \sigma\right)+X\left(e^{1} \sigma\right) \otimes\left(f^{1} \sigma\right)\right. \\
& \left.+Y\left(e^{2} \sigma\right) \otimes\left(f^{2} \sigma\right)+Z\left(e^{3} \sigma\right) \otimes\left(f^{3} \sigma\right)\right],
\end{aligned}
$$

where four-vectors $\left\{e^{\alpha}\right\}$ are defined by (19) and fulfill the condition (20), the same relations are fulfilled by four-vectors $\left\{f^{\alpha}\right\} ; N=\left(e^{0}\right)^{0}\left(f^{0}\right)^{0}+X\left(e^{1}\right)^{0}\left(f^{1}\right)^{0}+Y\left(e^{2}\right)^{0}\left(f^{2}\right)^{0}$ $+Z\left(e^{3}\right)^{0}\left(f^{3}\right)^{0}$ is a normalization factor.

Among the states (31) only four are pure, i.e., the states $\tilde{\rho}_{\mathrm{III}}(-1,-1,-1)$, $\tilde{\rho}_{\mathrm{III}}(-1,1,1), \tilde{\rho}_{\mathrm{III}}(1,-1,1)$, and $\tilde{\rho}_{\mathrm{III}}(1,1,-1)$. However, all of those states are connected with each other by transformations of the form (6). Moreover, only for the state $\tilde{\rho}_{\text {III }}(1,1,-1)$ the condition $1 \geq X \geq Y \geq|Z|$ is fulfilled. Therefore, all two-qubit pure entangled states can be generated from $\tilde{\rho}_{\mathrm{III}}(1,1,-1)$.

Now, let us determine transformations that leave $\Sigma_{\mathrm{III}}$ invariant under the action of the form given in Eq. (13). We assume that $X \neq-1, Y \neq-1, Z \neq-1$ (the case $X=Y=Z=-1$ corresponds to pure state, we have just considered it). Moreover, in all of the cases considered below, the little group contains the following discrete transformations:

$$
C \Sigma_{\mathrm{III}} C^{\mathrm{T}}=\Sigma_{\mathrm{III}}
$$

where $C$ has one of the following forms

$$
\begin{array}{ll}
C=\operatorname{diag}\{1,1,1,1\}, & C=\operatorname{diag}\{1,-1,-1,1\}, \\
C=\operatorname{diag}\{1,-1,1,-1\}, & C=\operatorname{diag}\{1,1,-1,-1\} .
\end{array}
$$

Now, let us determine transformations that leave $\Sigma_{\text {III }}$ invariant under the action of the form given in Eq. (13). The simplest method to determine a continuous little group is to find an infinitesimal form of the transformation, i.e., such infinitesimal antisymmetric matrices $A, \tilde{A}$ and such infinitesimal symmetric matrices $S, \tilde{S}$, that

$$
(I+A+S) \Sigma_{\mathrm{lII}}(I+\tilde{A}+\tilde{S})^{\mathrm{T}}=\Sigma_{\mathrm{III}}
$$

and

$$
A=\left(\begin{array}{ll}
0 & \mathbf{0}^{\mathrm{T}} \\
\mathbf{0} & \Omega
\end{array}\right), \quad S=\left(\begin{array}{cc}
0 & \mathbf{b}^{\mathrm{T}} \\
\mathbf{b} & \mathbf{0}
\end{array}\right)
$$

with $3 \times 3$ antisymmetric matrix $\Omega, 3 \times 3$ zero matrix $\mathbf{O}$ and with $\tilde{A}, \tilde{S}$ of analogous form.

Solving the appropriate equations, we receive the following little groups: (i) $X=$ $Y=Z=0$ : little group consists of arbitrary rotations on the left and arbitrary rotations on the right; (ii) $X=Y=Z<0$ : little group consists of all rotations on the left and the same rotations on the right (diagonal $\mathrm{SO}$ (3) group); (iii) $X, Y$, and $Z$ are pairwise distinct: little group is trivial and contains only operations given in Eq. (35); (iv) $X=Y \neq Z, Z=-1$ : in this case we have two subcases: (iva) $X=Y=0$ : little group consists of Lorentz boosts in the direction of the third axis on the left and 
the same boosts on the right and arbitrary rotations in the $X-Y$ plane on the left and arbitrary rotations in the same plane on the right, (ivb) $X=Y \neq 0$ : little group consists of Lorentz boosts in the direction of the third axis and rotations in the $X-Y$ plane on the left and the same boosts and rotations on the right; (v) $X=Y \neq Z$, $Z \neq-1$ : in this case we also have two subcases: (va) $X=Y=0$ : little group consists of rotations in the $X-Y$ plane on the left and arbitrary rotations in the same plane on the right, (vb) $X=Y \neq 0$ : little group consists of rotations in the $X-Y$ plane on the left and the same rotations on the right.

\subsection{The orbit generated by the state $\Sigma_{\mathrm{IV}}[\mathrm{Eq} \cdot(12)]$}

The corresponding density matrix has the following form

$$
\tilde{\rho}_{\mathrm{IV}}(x, y)=\frac{1}{2}\left(\begin{array}{cccc}
2 y & 0 & 0 & 0 \\
0 & 1-y & x & 0 \\
0 & x & 1-y & 0 \\
0 & 0 & 0 & 0
\end{array}\right)
$$

where $0 \leq x<1,0<y<1-x$. This is a mixed state, and its concurrence is equal to

$$
C\left(\tilde{\rho}_{\mathrm{IV}}(x, y)\right)=x .
$$

All states generated from $\tilde{\rho}_{\text {IV }}$ via the transformation (6) can be written in the following form

$$
\begin{aligned}
\tilde{\rho}_{\mathrm{IV}} \mapsto \rho_{\mathrm{IV}}= & \frac{1}{4 N}\left[\left(e^{0} \sigma\right) \otimes\left(f^{0} \sigma\right)+x\left(e^{1} \sigma\right) \otimes\left(f^{1} \sigma\right)+x\left(e^{2} \sigma\right) \otimes\left(f^{2} \sigma\right)\right. \\
& \left.+(2 y-1)\left(e^{3} \sigma\right) \otimes\left(f^{3} \sigma\right)+y\left(e^{0} \sigma\right) \otimes\left(f^{3} \sigma\right)+y\left(e^{3} \sigma\right) \otimes\left(f^{0} \sigma\right)\right],
\end{aligned}
$$

where four-vectors $\left\{e^{\alpha}\right\}$ are defined by (19) and fulfill the condition (20), and the same relations are fulfilled by four-vectors $\left\{f^{\alpha}\right\} ; N=\left(e^{0}\right)^{0}\left(f^{0}\right)^{0}+x\left[\left(e^{1}\right)^{0}\left(f^{1}\right)^{0}\right.$ $\left.+\left(e^{2}\right)^{0}\left(f^{2}\right)^{0}\right]+(2 y-1)\left(e^{3}\right)^{0}\left(f^{3}\right)^{0}+y\left[\left(e^{0}\right)^{0}\left(f^{3}\right)^{0}+\left(e^{3}\right)^{0}\left(f^{0}\right)^{0}\right]$ is the normalization factor.

Let us comment the relationship between our parametrization of the generating matrix (38) and the corresponding form of rank-deficient generating family given in [4]. First of all notice that matrices from this rank-deficient family in general are not positive semidefinite. These matrices are positive semidefinite provided that the parameters fulfill appropriate conditions (see Eq. 3). In such a case the rank-deficient density matrices given in [4] form a 3D manifold which belongs to the orbit defined by (40) (except some special cases belonging to the orbits generated from $(8,9)$ ). However, the generating set introduced in [4] is not a minimal one. Indeed, our set of states defined in (38) form a 2D manifold belonging to the same orbit. We proved that this is a minimal generating set (see "Appendix 2").

For the state (38), we can also calculate a quantum discord [2,7] because this state may have non-classical correlations beyond quantum entanglement. The concept of 
quantum discord is based on the idea that two equivalent ways of calculating mutual information in classical information theory give different results when generalized to quantum ground. Namely, let us consider two discrete random variables $A$ and $B$ with values $a$ and $b$ occurring with probabilities $p_{a}$ and $p_{b}$, respectively. The classical mutual information describing the total amount of correlations between random variables $A, B$ is defined as

$$
\mathscr{I}_{c l}(A: B)=H(A)+H(B)-H(A, B),
$$

where $H(A)=-\sum_{a} p_{a} \log p_{a}$ denotes the Shannon entropy and analogous formulas hold for $H(B)$ and for the joint entropy $H(A, B)$ (all logarithms are base 2). For classical random variables, a conditional probability $p_{a \mid b}$ is equal to $p_{a \mid b}=p_{a b} / p_{b}$. Therefore, the classical mutual information can be written in an equivalent form

$$
\mathscr{J}_{c l}(A: B)=H(A)-H(A \mid B)
$$

where the conditional entropy $H(A \mid B)=H(A, B)-H(B)$.

In the quantum case, we consider a two-partite system described by a density matrix $\rho$ with subsystems $A, B$ with density matrices $\rho_{A}=\operatorname{Tr}_{B}(\rho), \rho_{B}=\operatorname{Tr}_{A}(\rho)$. The formula (41) is generalized in a straightforward way to

$$
\mathscr{I}(A: B)=S\left(\rho_{A}\right)+S\left(\rho_{B}\right)-S(\rho)
$$

where $S\left(\rho_{A}\right)=-\operatorname{Tr}\left(\rho_{A} \log \rho_{A}\right)$ is the von Neumann entropy of a subsystem $A$ and analogous formulas holds for $S\left(\rho_{B}\right), S(\rho)$. On the other hand, the quantum generalization of (42) cannot be done in a straightforward way, i.e.,

$$
\mathscr{J}(A: B)=S\left(\rho_{A}\right)-S(\rho \mid B)
$$

since the conditional quantum entropy $S(\rho \mid B)$ depends on an observable we have measured on $B$. Recall that after a non-selective projective measurement on $B$, the system is transformed into a statistical ensemble $\left\{p_{k}, \rho_{k}\right\}, k=1,2$, where

$$
p_{1}=\operatorname{Tr}\left[\left(I_{A} \otimes \Pi_{B}\right) \rho\right], \quad \rho_{1}=\frac{\left(I_{A} \otimes \Pi_{B}\right) \rho\left(I_{A} \otimes \Pi_{B}\right)}{p_{1}},
$$

and similar formulas for $p_{2}, \rho_{2}$ with $\Pi_{B}$ replaced with $\Pi_{B}^{\perp}$ (here, $\Pi_{B}$ and $\Pi_{B}^{\perp}$ are orthogonal rank-one projectors). Therefore, we define the quantum version of (42) by taking the least disturbing measurement, i.e.,

$$
\mathscr{C}(A: B)=\max _{\left\{\Pi_{B}\right\}} \mathscr{J}(A: B)=S\left(\rho_{A}\right)-\min _{\left\{\Pi_{B}\right\}} S\left(\rho \mid\left\{\Pi_{B}\right\}\right),
$$

where

$$
S\left(\rho \mid\left\{\Pi_{B}\right\}\right)=p_{1} S\left(\rho_{1}\right)+p_{2} S\left(\rho_{2}\right)
$$

(compare (45)). Consequently, the quantum discord describing the amount of genuinely quantum correlations is defined as 


$$
\mathscr{D}(A: B)=\mathscr{I}(A: B)-\mathscr{C}(A: B)=S\left(\rho_{B}\right)-S(\rho)+\min _{\left\{\Pi_{B}\right\}} S\left(\rho \mid\left\{\Pi_{B}\right\}\right) .
$$

The quantum discord $\mathscr{D}(A: B)$ can be seen as the minimal amount of correlations which are lost when the non-selective von Neumann projective measurement is taken on the system $B[8,9]$. Moreover, the quantum discord $\mathscr{D}(A: B)$ is a lower bound for the global quantum correlations present in a bipartite state $\rho$ [9].

Since evaluation of quantum discord involves a complicated optimization procedure, the analytical expressions for quantum discord are known only for two-qubit Bell-diagonal states [10], for seven-parameter two-qubit $X$ states [11] (not always correct exactly, but approximately correct with a very small absolute error [12]), for two-mode Gaussian states [13,14], for a class of two-qubit states with parallel nonzero Bloch vectors [15] and for two-qubit Werner and isotropic states [16]. Despite this fact, quantum discord has been studied in different contexts [2].

Moreover, recently it has been shown that computing quantum discord is an NPcomplete problem [17].

However, we present here the calculation of the discord of the state (38) since our method of calculation is simpler. First of all, eigenvalues of (38) are equal to:

$$
\lambda_{0}=0, \quad \lambda_{1}=y, \quad \lambda_{2}=\frac{1}{2}(1-y-x), \quad \lambda_{3}=\frac{1}{2}(1-y+x),
$$

and

$$
\rho_{A}=\rho_{B}=\frac{1}{2}\left(I+y \sigma_{3}\right)
$$

Therefore

$$
S(\rho)=1-\frac{1}{2}[2 y \log (2 y)+(1-y-x) \log (1-y-x)+(1-y+x) \log (1-y+x)],
$$

and

$$
S\left(\rho_{A}\right)=S\left(\rho_{B}\right)=1-\frac{1}{2}[(1-y) \log (1-y)+(1+y) \log (1+y)] .
$$

Now, to calculate the quantum conditional entropy, we need the most general projector $\Pi_{B}$. Such a projector can be parametrized as follows

$$
\Pi_{B} \equiv \Pi(\mathbf{e})=\frac{1}{2}(I+\mathbf{e} \cdot \boldsymbol{\sigma}),
$$

where $\mathbf{e}$ is an arbitrary unit vector $\left(\mathbf{e}^{2}=1\right)$. Notice that in $[11,15,18]$ (and also, e.g., in [10]), the more complicated parametrization of this projector was used. It holds

$$
\Pi_{B}^{\perp}=\Pi(-\mathbf{e}) .
$$

Therefore

$$
\begin{aligned}
S\left(\rho \mid\left\{\Pi_{B}\right\}\right)= & p(\mathbf{e}) S\left(\frac{\operatorname{Tr}_{B}[(I \otimes \Pi(\mathbf{e})) \rho(I \otimes \Pi(\mathbf{e}))]}{p(\mathbf{e})}\right) \\
& +p(-\mathbf{e}) S\left(\frac{\operatorname{Tr}_{B}[(I \otimes \Pi(-\mathbf{e})) \rho(I \otimes \Pi(-\mathbf{e}))]}{p(-\mathbf{e})}\right) .
\end{aligned}
$$


Simple calculation shows that in our case

$$
p(\mathbf{e})=\operatorname{Tr}[(I \otimes \Pi(\mathbf{e})) \rho]=\frac{1}{2}\left(1+e_{3} y\right)
$$

and

$$
p(-\mathbf{e})=\operatorname{Tr}[(I \otimes \Pi(-\mathbf{e})) \rho]=\frac{1}{2}\left(1-e_{3} y\right)
$$

Furthermore

$$
\frac{\operatorname{Tr}_{B}[(I \otimes \Pi(\mathbf{e})) \rho(I \otimes \Pi(\mathbf{e}))]}{p(\mathbf{e})}=\frac{1}{2}(I+\mathbf{r}(\mathbf{e}) \cdot \boldsymbol{\sigma})
$$

with

$$
\mathbf{r}(\mathbf{e})=\left(\frac{x e_{1}}{1+e_{3} y}, \frac{x e_{2}}{1+e_{3} y}, \frac{y+(2 y-1) e_{3}}{1+e_{3} y}\right) .
$$

Eigenvalues of $\operatorname{Tr}_{B}[(I \otimes \Pi(\mathbf{e})) \rho(I \otimes \Pi(\mathbf{e}))] / p(\mathbf{e})$ and $\operatorname{Tr}_{B}[(I \otimes \Pi(-\mathbf{e})) \rho(I \otimes$ $\Pi(-\mathbf{e}))] / p(-\mathbf{e})$ are equal to

$$
\lambda^{ \pm}(\mathbf{e})=\frac{1}{2}\left[1 \pm \frac{\sqrt{\left(1-e^{2}\right) x^{2}+[y+(2 y-1) e]^{2}}}{1+e y}\right]
$$

and

$$
\lambda^{ \pm}(-\mathbf{e})=\frac{1}{2}\left[1 \pm \frac{\sqrt{\left(1-e^{2}\right) x^{2}+[y-(2 y-1) e]^{2}}}{1-e y}\right]
$$

respectively. $S(\rho \mid \Pi(\mathbf{e}))$ and $S(\rho \mid \Pi(-\mathbf{e}))$ depend only on $e_{3}$, so, to simplify the notation, we have put $e=e_{3}$. Therefore, we have to minimize, with respect to $e \in\langle-1,1\rangle$, the following function

$$
\begin{aligned}
S(\rho \mid\{\Pi(\mathbf{e})\})= & -\frac{1}{2}(1+e y)\left[\lambda^{+}(\mathbf{e}) \log \lambda^{+}(\mathbf{e})+\lambda^{-}(\mathbf{e}) \log \lambda^{-}(\mathbf{e})\right] \\
& -\frac{1}{2}(1-e y)\left[\lambda^{+}(-\mathbf{e}) \log \lambda^{+}(-\mathbf{e})+\lambda^{-}(-\mathbf{e}) \log \lambda^{-}(-\mathbf{e})\right] .
\end{aligned}
$$

The value of $e$ for which the minimum is achieved depends on $x$ and $y$. The function $S(\rho \mid\{\Pi(\mathbf{e})\})$ is symmetric with respect to $e=0$, while its derivative (with respect to $e$ ) is antisymmetric. Therefore, $\left.\partial_{e} S(\rho \mid\{\Pi(\mathbf{e})\})\right|_{e=0}=0$. The minimum can be achieved only for $e=0$ or on the border for $e= \pm 1$. Thus, the triangle $0 \leq x<1,0<y<1-x$ is divided into two regions. In the first region, the minimum is achieved for $e=0$ (region I in Fig. 1), while in the second region, the minimum is achieved for $e= \pm 1$ (region II in Fig. 1). Furthermore, the border between these two regions is described by the equation

$$
\begin{aligned}
& \left(1-\sqrt{x^{2}+y^{2}}\right) \log \left(1-\sqrt{x^{2}+y^{2}}\right)+(1+y) \log (1+y)-(1-y) \log (1-y) \\
& +\left(1+\sqrt{x^{2}+y^{2}}\right) \log \left(1+\sqrt{x^{2}+y^{2}}\right)-2 y \log (2 y)=2 .
\end{aligned}
$$




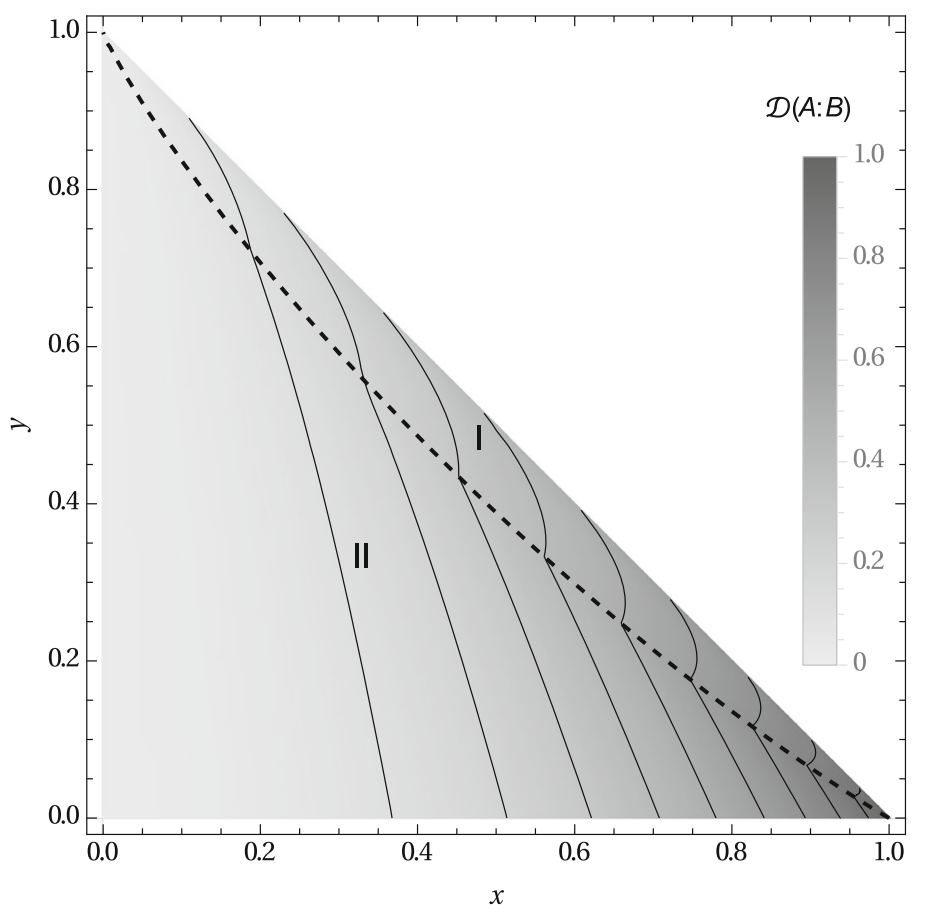

Fig. 1 Density plot of the discord (65) in the parameter space $(0 \leq x<1,0<y<1-x)$. I and II denote regions in which $S(\rho \mid\{\Pi(\mathbf{e})\})$ given in (62) achieves minimum for different values of $e$. The border between regions (dashed line) is described by Eq. (63). Solid lines represent level curves of the discord

The minima in corresponding regions are equal to

$$
\begin{aligned}
S_{\mathrm{I}}(e=0)= & 1-\frac{1}{2}\left[\left(1-\sqrt{x^{2}+y^{2}}\right) \log \left(1-\sqrt{x^{2}+y^{2}}\right)\right. \\
& \left.+\left(1+\sqrt{x^{2}+y^{2}}\right) \log \left(1+\sqrt{x^{2}+y^{2}}\right)\right]
\end{aligned}
$$

and

$$
S_{\mathrm{II}}(e= \pm 1)=-\frac{1}{2}[(1-y) \log (1-y)-(1+y) \log (1+y)+2 y \log (2 y)]
$$

respectively. Evidently, $S_{\text {I }}$ and $S_{\text {II }}$ are equal on the curve given in Eq. (63).

Therefore, using the definition $(48)$ and $(51,52)$ we can easily determine quantum discord in both regions, I and II:

$$
\begin{aligned}
\mathscr{D}_{\mathrm{I}}= & 1+\frac{1}{2}[2 y \log (2 y)+(1-y-x) \log (1-y-x)+(1-y+x) \log (1-y+x) \\
& -(1-y) \log (1-y)-(1+y) \log (1+y)-\left(1-\sqrt{x^{2}+y^{2}}\right) \log \left(1-\sqrt{x^{2}+y^{2}}\right) \\
& \left.-\left(1+\sqrt{x^{2}+y^{2}}\right) \log \left(1+\sqrt{x^{2}+y^{2}}\right)\right]
\end{aligned}
$$




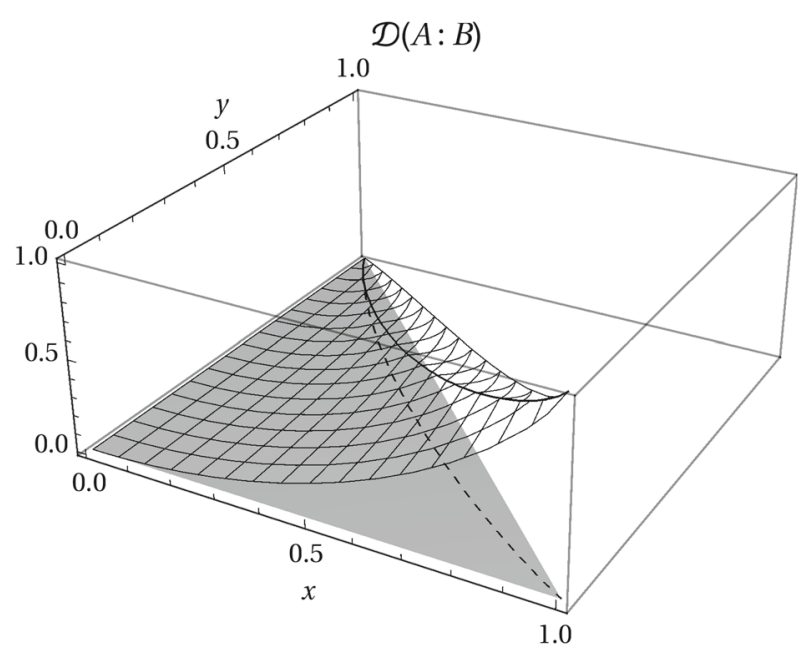

Fig. 2 Quantum discord (65) of the state (38) as a function of $x$ and $y$ where $0 \leq x<1,0<y<1-x$. Dashed line corresponds to the border between regions I and II from Fig. 1

and

$\mathscr{D}_{\mathrm{II}}=\frac{1}{2}(1-y-x) \log (1-y-x)+\frac{1}{2}(1-y+x) \log (1-y+x)-(1-y) \log (1-y)$.

In Fig. 2, we have depicted the quantum discord as a function of $x$ and $y$.

Applying the same methods as in the previous subsection, we arrive at the following form of little group of $\Sigma_{\mathrm{IV}}$ : (i) when $x=0$, little group consists of arbitrary rotations around the third axis on the left and arbitrary rotations around the third axis on the right and (ii) when $x \neq 0$, little group consists of arbitrary rotations around the third axis on the left and the same rotations on the right (diagonal $\mathrm{SO}(2)$ group).

\section{Conclusions}

We have thoroughly discussed the classification of two-qubit states with respect to transformations (6). This problem for the first time was discussed in [4], where it was shown that each two-qubit state can be obtained with the help of transformations (6) from one of the two canonical families of states. One of these families corresponds to Bell-diagonal states, while the other one is a three-parameter family of rank-deficient states. We have shown that the latter family of states should be replaced by three fixed states and a two-parameter family of states. We have shown also that this set of states is a minimal generating set with respect to transformations (6). We have exhaustively discussed this two-parameter family of states. In particular, we have found an explicit and very simple parametrization guaranteeing positive semidefiniteness of states from this two-parameter family of states. We have also calculated the Wootters concurrence and quantum discord for these states. It is worth noting that for these states the quantum discord is zero if and only if the state is separable. 
Moreover, we have also found a new general parametrization of all two-qubit states generated from the canonical families, where sets of (pseudo)orthogonal four-vectors (frames) were used. An advantage of our parametrization lies in fact that the standard conditions for positive semidefiniteness of density matrices [5] are equivalent to (pseudo)orthogonality conditions for four-vectors serving as parameters (and appropriate conditions for parameters of the corresponding canonical family of states).

Acknowledgments J. R. thanks Marek Kuś and Karol Życzkowski for interesting discussion.

Open Access This article is distributed under the terms of the Creative Commons Attribution 4.0 International License (http://creativecommons.org/licenses/by/4.0/), which permits unrestricted use, distribution, and reproduction in any medium, provided you give appropriate credit to the original author(s) and the source, provide a link to the Creative Commons license, and indicate if changes were made.

\section{Appendix 1: Algebraic prerequisites}

We use the results from [19]. Real Jordan blocks are defined as follows:

$$
J_{m}(\lambda)=\left(\begin{array}{ccccc}
\lambda & 1 & 0 & \ldots & 0 \\
0 & \lambda & 1 & \ldots & 0 \\
\vdots & \vdots & & & 0 \\
\vdots & \vdots & & \lambda & 1 \\
0 & 0 & \vdots & 0 & \lambda
\end{array}\right)
$$

$J_{m}(\lambda)$ is a $m \times m$ real matrix, $\lambda \in \mathbb{R}$; and

$$
J_{2 m}(\lambda \pm i \mu)=\left(\begin{array}{ccccc}
j(\lambda, \mu) & I_{2} & \mathbf{O} & \ldots & \mathbf{O} \\
\mathbf{O} & j_{(\lambda, \mu)} & I_{2} & \ldots & \mathbf{O} \\
\vdots & & & & \\
\mathbf{O} & \mathbf{O} & \ldots & j_{(\lambda, \mu)} & I_{2} \\
\mathbf{O} & \mathbf{O} & \ldots & \mathbf{O} & j_{(\lambda, \mu)}
\end{array}\right)
$$

where

$$
j_{(\lambda, \mu)}=\left(\begin{array}{cc}
\lambda & \mu \\
-\mu & \lambda
\end{array}\right), \quad \mathbf{O}=\left(\begin{array}{ll}
0 & 0 \\
0 & 0
\end{array}\right),
$$

$I_{2}$ is a $2 \times 2$ identity matrix. $J_{2 m}(\lambda \pm i \mu)$ is a $2 m \times 2 m$ real matrix, $\lambda, \mu \in \mathbb{R}, \mu \neq 0$.

Theorem 2 (Real Jordan form) For any real, $n \times n$ matrix $A$, there exists an invertible real matrix $S$ such that

$$
S A S^{-1}=J,
$$

where $J$ (a real Jordan form of $A$ ) is a block diagonal matrix and has the form

$$
J=J_{m_{1}}\left(\lambda_{1}\right) \oplus J_{m_{2}}\left(\lambda_{2}\right) \oplus \cdots \oplus J_{m_{r}}\left(\lambda_{r}\right) \oplus J_{2 m_{r+1}}\left(\lambda_{r+1} \pm i \mu_{r+1}\right) \oplus \cdots \oplus J_{2 m_{q}}\left(\lambda_{q} \pm i \mu_{q}\right),
$$


where the $\lambda_{j}$ are real and the $\mu_{j}$ are real and positive. $\lambda_{j}$ for $1 \leq j \leq r$ and $\lambda_{k} \pm i \mu_{k}$ for $r+1 \leq k \leq q$ are eigenvalues of the matrix A. The size of a Jordan block $J_{j}$ is determined by the multiplicity of the corresponding eigenvalue in the characteristic polynomial of $A$.

For a matrix $J$ given in Eq. (70), we define a matrix

$$
P_{\varepsilon, J}=\varepsilon_{1} P_{1} \oplus \cdots \oplus \varepsilon_{r} P_{r} \oplus P_{r+1} \oplus \cdots \oplus P_{q}
$$

where $\varepsilon=\left(\varepsilon_{1}, \ldots, \varepsilon_{r}\right)$ and $\varepsilon_{j}= \pm 1$ for each $j$. Matrices $P_{j}$ have the following form

$$
P_{j}=\left(\begin{array}{ccccc}
0 & 0 & \ldots & 0 & 1 \\
0 & 0 & \ldots & 1 & 0 \\
\vdots & & & & \vdots \\
0 & 1 & \ldots & 0 & 0 \\
1 & 0 & \ldots & 0 & 0
\end{array}\right)
$$

and are of the size equal to that of $J_{j}$.

The following theorem (theorem 6.1.5 from [19]) holds:

Theorem 3 A pair $(A, H)$ of real matrices, where $H$ is real and invertible and $A=$ $H^{-1} A^{\mathrm{T}} H$ is $r$-unitary similar to a pair $\left(J, P_{\varepsilon, J}\right)$, where $J$ is a real Jordan form of $A$, and $P_{\varepsilon, J}$ is given in Eq. (71). The signs $\varepsilon_{j}$ are determined uniquely by $(A, H)$ up to permutation of signs in the blocks of $P_{\varepsilon, J}$ corresponding to the Jordan blocks of $J$ with the same real eigenvalue and the same size.

A pair $(A, H)$ is said to be $r$-unitary similar to a pair $\left(J, P_{\varepsilon, J}\right)$ iff there exists a non-degenerate real matrix $S$ such that

$$
A=S^{-1} J S, \quad H=S^{\mathrm{T}} P_{\varepsilon, J} S
$$

\section{Appendix 2: Canonical Form of Two-Qubit States}

In this Appendix, we give a detailed proof of the Theorem 1.

Each two-qubit density matrix can be written in the form given in Eq. (2). Now, define a real matrix

$$
A=\eta R \eta R^{\mathrm{T}},
$$

where $\eta=\operatorname{diag}(+,-,-,-)$ is the Minkowski metric tensor. One can easily check that it holds

$$
A=\eta^{-1} A^{\mathrm{T}} \eta=\eta A^{\mathrm{T}} \eta .
$$

Therefore, Theorem 3 implies that the pair $(A, \eta)$ is $r$-unitary equivalent to a pair $\left(J, P_{\varepsilon, J}\right)$, that is, there exists a non-degenerate, real matrix $S$ such that

$$
A=S^{-1} J S, \quad \eta=S^{\mathrm{T}} P_{\varepsilon, J} S,
$$


where $J$ is a real Jordan form of $A$ and $P_{\varepsilon, J}$ is given in Eq. (71). Below we give all possible forms of $J$ and corresponding $P_{\varepsilon, J}$ that are $r$-unitary equivalent to $(A, \eta)$.

$$
\begin{aligned}
& J_{(1)}=\left(\begin{array}{cccc}
\lambda & 1 & 0 & 0 \\
0 & \lambda & 1 & 0 \\
0 & 0 & \lambda & 1 \\
0 & 0 & 0 & \lambda
\end{array}\right), \quad P_{\varepsilon, J_{(1)}}=\left(\begin{array}{cccc}
0 & 0 & 0 & \varepsilon \\
0 & 0 & \varepsilon & 0 \\
0 & \varepsilon & 0 & 0 \\
\varepsilon & 0 & 0 & 0
\end{array}\right) \\
& J_{(2)}=\left(\begin{array}{cccc}
\lambda_{1} & 1 & 0 & 0 \\
0 & \lambda_{1} & 1 & 0 \\
0 & 0 & \lambda_{1} & 0 \\
0 & 0 & 0 & \lambda_{2}
\end{array}\right), \quad P_{\varepsilon, J_{(2)}}=\left(\begin{array}{cccc}
0 & 0 & \varepsilon_{1} & 0 \\
0 & \varepsilon_{1} & 0 & 0 \\
\varepsilon_{1} & 0 & 0 & 0 \\
0 & 0 & 0 & \varepsilon_{2}
\end{array}\right) ; \\
& J_{(2)}^{\prime}=\left(\begin{array}{cccc}
\lambda_{1} & 0 & 0 & 0 \\
0 & \lambda_{2} & 1 & 0 \\
0 & 0 & \lambda_{2} & 1 \\
0 & 0 & 0 & \lambda_{2}
\end{array}\right), \quad P_{\varepsilon, J_{(2)}^{\prime}}=\left(\begin{array}{cccc}
\varepsilon_{1} & 0 & 0 & 0 \\
0 & 0 & 0 & \varepsilon_{2} \\
0 & 0 & \varepsilon_{2} & 0 \\
0 & \varepsilon_{2} & 0 & 0
\end{array}\right) \text {; } \\
& J_{(3)}=\left(\begin{array}{cccc}
\lambda_{1} & 1 & 0 & 0 \\
0 & \lambda_{1} & 0 & 0 \\
0 & 0 & \lambda_{2} & 1 \\
0 & 0 & 0 & \lambda_{2}
\end{array}\right), \quad P_{\varepsilon, J_{(3)}}=\left(\begin{array}{cccc}
0 & \varepsilon_{1} & 0 & 0 \\
\varepsilon_{1} & 0 & 0 & 0 \\
0 & 0 & 0 & \varepsilon_{2} \\
0 & 0 & \varepsilon_{2} & 0
\end{array}\right) \text {; } \\
& J_{(4)}=\left(\begin{array}{cccc}
\lambda_{1} & 1 & 0 & 0 \\
0 & \lambda_{1} & 0 & 0 \\
0 & 0 & \lambda_{2} & 0 \\
0 & 0 & 0 & \lambda_{3}
\end{array}\right), \quad P_{\varepsilon, J_{(4)}}=\left(\begin{array}{cccc}
0 & \varepsilon_{1} & 0 & 0 \\
\varepsilon_{1} & 0 & 0 & 0 \\
0 & 0 & \varepsilon_{2} & 0 \\
0 & 0 & 0 & \varepsilon_{3}
\end{array}\right) \text {; } \\
& J_{(4)}^{\prime}=\left(\begin{array}{cccc}
\lambda_{1} & 0 & 0 & 0 \\
0 & \lambda_{2} & 0 & 0 \\
0 & 0 & \lambda_{3} & 1 \\
0 & 0 & 0 & \lambda_{3}
\end{array}\right), \quad P_{\varepsilon, J_{(4)}^{\prime}}=\left(\begin{array}{cccc}
\varepsilon_{1} & 0 & 0 & 0 \\
0 & \varepsilon_{2} & 0 & 0 \\
0 & 0 & 0 & \varepsilon_{3} \\
0 & 0 & \varepsilon_{3} & 0
\end{array}\right) ; \\
& J_{(4)}^{\prime \prime}=\left(\begin{array}{cccc}
\lambda_{1} & 0 & 0 & 0 \\
0 & \lambda_{2} & 1 & 0 \\
0 & 0 & \lambda_{2} & 0 \\
0 & 0 & 0 & \lambda_{3}
\end{array}\right), \quad P_{\varepsilon, J_{(4)}^{\prime \prime}}=\left(\begin{array}{cccc}
\varepsilon_{1} & 0 & 0 & 0 \\
0 & 0 & \varepsilon_{2} & 0 \\
0 & \varepsilon_{2} & 0 & 0 \\
0 & 0 & 0 & \varepsilon_{3}
\end{array}\right) \text {; } \\
& J_{(5)}=\left(\begin{array}{cccc}
\lambda_{1} & 0 & 0 & 0 \\
0 & \lambda_{2} & 0 & 0 \\
0 & 0 & \lambda_{3} & 0 \\
0 & 0 & 0 & \lambda_{4}
\end{array}\right), \quad P_{\varepsilon, J_{(5)}}=\left(\begin{array}{cccc}
\varepsilon_{1} & 0 & 0 & 0 \\
0 & \varepsilon_{2} & 0 & 0 \\
0 & 0 & \varepsilon_{3} & 0 \\
0 & 0 & 0 & \varepsilon_{4}
\end{array}\right) ; \\
& J_{(6)}=\left(\begin{array}{cccc}
\sigma & \tau & 1 & 0 \\
-\tau & \sigma & 0 & 1 \\
0 & 0 & \sigma & \tau \\
0 & 0 & -\tau & \sigma
\end{array}\right), \quad P_{\varepsilon, J_{(6)}}=\left(\begin{array}{cccc}
0 & 0 & 0 & 1 \\
0 & 0 & 1 & 0 \\
0 & 1 & 0 & 0 \\
1 & 0 & 0 & 0
\end{array}\right) \text {; }
\end{aligned}
$$




$$
\begin{aligned}
& J_{(7)}=\left(\begin{array}{cccc}
\sigma & \tau & 0 & 0 \\
-\tau & \sigma & 0 & 0 \\
0 & 0 & \sigma^{\prime} & \tau^{\prime} \\
0 & 0 & -\tau^{\prime} & \sigma^{\prime}
\end{array}\right), \quad P_{\varepsilon, J_{(7)}}=\left(\begin{array}{cccc}
0 & 1 & 0 & 0 \\
1 & 0 & 0 & 0 \\
0 & 0 & 0 & 1 \\
0 & 0 & 1 & 0
\end{array}\right) ; \\
& J_{(8)}=\left(\begin{array}{cccc}
\sigma & \tau & 0 & 0 \\
-\tau & \sigma & 0 & 0 \\
0 & 0 & \lambda & 1 \\
0 & 0 & 0 & \lambda
\end{array}\right), \quad P_{\varepsilon, J_{(8)}}=\left(\begin{array}{cccc}
0 & 1 & 0 & 0 \\
1 & 0 & 0 & 0 \\
0 & 0 & 0 & \varepsilon \\
0 & 0 & \varepsilon & 0
\end{array}\right) \text {; } \\
& J_{(8)}^{\prime}=\left(\begin{array}{cccc}
\lambda & 1 & 0 & 0 \\
0 & \lambda & 0 & 0 \\
0 & 0 & \sigma & \tau \\
0 & 0 & -\tau & \sigma
\end{array}\right), \quad P_{\varepsilon, J_{(8)}^{\prime}}=\left(\begin{array}{cccc}
0 & \varepsilon & 0 & 0 \\
\varepsilon & 0 & 0 & 0 \\
0 & 0 & 0 & 1 \\
0 & 0 & 1 & 0
\end{array}\right) ; \\
& J_{(9)}=\left(\begin{array}{cccc}
\sigma & \tau & 0 & 0 \\
-\tau & \sigma & 0 & 0 \\
0 & 0 & \lambda_{1} & 0 \\
0 & 0 & 0 & \lambda_{2}
\end{array}\right), \quad P_{\varepsilon, J_{(9)}}=\left(\begin{array}{cccc}
0 & 1 & 0 & 0 \\
1 & 0 & 0 & 0 \\
0 & 0 & \varepsilon_{1} & 0 \\
0 & 0 & 0 & \varepsilon_{2}
\end{array}\right) \text {; } \\
& J_{(9)}^{\prime}=\left(\begin{array}{cccc}
\lambda_{1} & 0 & 0 & 0 \\
0 & \sigma & \tau & 0 \\
0 & -\tau & \sigma & 0 \\
0 & 0 & 0 & \lambda_{2}
\end{array}\right), \quad P_{\varepsilon, J_{(9)}^{\prime}}=\left(\begin{array}{cccc}
\varepsilon_{1} & 0 & 0 & 0 \\
0 & 0 & 1 & 0 \\
0 & 1 & 0 & 0 \\
0 & 0 & 0 & \varepsilon_{2}
\end{array}\right) \text {; } \\
& J_{(9)}^{\prime \prime}=\left(\begin{array}{cccc}
\lambda_{1} & 0 & 0 & 0 \\
0 & \lambda_{2} & 0 & 0 \\
0 & 0 & \sigma & \tau \\
0 & 0 & -\tau & \sigma
\end{array}\right), \quad P_{\varepsilon, J_{(9)}^{\prime \prime}}=\left(\begin{array}{cccc}
\varepsilon_{1} & 0 & 0 & 0 \\
0 & \varepsilon_{2} & 0 & 0 \\
0 & 0 & 0 & 1 \\
0 & 0 & 1 & 0
\end{array}\right) \text {. }
\end{aligned}
$$

In Eqs. (77-91), $\lambda_{i} \in \mathbb{R}, \sigma, \tau, \sigma^{\prime}, \tau^{\prime} \in \mathbb{R}, \tau, \tau^{\prime}>0$ and $\varepsilon_{i}= \pm 1$.

Now, from Eq. (76), it follows that $P_{\varepsilon, J}$ is symmetric. Therefore, it can be diagonalized by the similarity transformation with the help of orthogonal matrix. That is, for every $P_{\varepsilon, J}$ there exists such a diagonal matrix $D$ and orthogonal matrix $\tilde{\Omega}$ that

$$
P_{\varepsilon, J}=\tilde{\Omega}^{\mathrm{T}} D \tilde{\Omega}
$$

Each $P_{\varepsilon, J}$ is congruent with $\eta$. Thus, inserting necessary permutations, we can write

$$
P_{\varepsilon, J}=\Omega^{\mathrm{T}} \eta \Omega \text { with } \Omega^{\mathrm{T}} \Omega=I
$$

Sylvester's law of inertia implies that some of the possibilities from (77-91) are excluded (signature of a bilinear form determined by the corresponding $P_{\varepsilon, J}$ is different from the signature of the form determined by $\eta$ ). In this way, we exclude cases with numbers (1), (3), (6), (7), (8). 
Notice that $S^{\mathrm{T}} \Omega^{\mathrm{T}}=\Lambda$ is a Lorentz transformation because using Eqs. $(76,93)$, we have

$$
\begin{aligned}
S^{\mathrm{T}} \Omega^{\mathrm{T}} \eta\left(S^{\mathrm{T}} \Omega^{\mathrm{T}}\right)^{\mathrm{T}} & =S^{\mathrm{T}}\left(\Omega^{\mathrm{T}} \eta \Omega\right) S \\
& =S^{\mathrm{T}} P_{\varepsilon, J} S \\
& =\eta .
\end{aligned}
$$

Therefore, Eqs. $(74,76,93)$ implies

$$
R \eta R^{\mathrm{T}}=\Lambda \eta \Omega J \Omega^{\mathrm{T}} \Lambda^{\mathrm{T}} .
$$

Now, we want to determine matrix $\Sigma$ such that

$$
\Sigma \eta \Sigma^{\mathrm{T}}=\eta \Omega J \Omega^{\mathrm{T}}
$$

For such a matrix, we have

$$
R \eta R^{\mathrm{T}}=\Lambda \Sigma \eta \Sigma^{\mathrm{T}} \Lambda^{\mathrm{T}}
$$

Thus

$$
R=\Lambda \Sigma \tilde{\Lambda}^{\mathrm{T}},
$$

where $\Lambda, \tilde{\Lambda}$ are Lorentz transformations. Thus, we want to determine the simplest form of $\Sigma$ from Eq. (96) in all of the cases given in Eqs. (77-91). In some of these cases, e.g., (2) and (2)', Jordan forms differ by the permutation of Jordan blocks. For example

$$
J_{(2)}^{\prime}=X J_{(2)} X^{\mathrm{T}}, \quad X X^{\mathrm{T}}=I,
$$

where $X$ is a permutation matrix. If follows that

$$
P_{\varepsilon, J_{(2)}^{\prime}}=S P_{\varepsilon, J_{(2)}} S^{\mathrm{T}}
$$

We have

$$
P_{\varepsilon, J_{(2)}^{\prime}}=\Omega_{(2)}^{\prime \mathrm{T}} \eta \Omega_{(2)}^{\prime} \quad \text { and } \quad P_{\varepsilon, J_{(2)}}=\Omega_{(2)}^{\mathrm{T}} \eta \Omega_{(2)} .
$$

Therefore, we can take $\Omega_{(2)}=\Omega_{(2)}^{\prime} X$ and consequently

$$
\begin{aligned}
\Sigma_{2} \eta \Sigma_{2}^{\mathrm{T}} & =\eta \Omega_{2} J_{(2)} \Omega_{2}^{\mathrm{T}} \\
& =\eta \Omega_{(2)}^{\prime} X J_{(2)} X^{\mathrm{T}} \Omega_{(2)}^{\mathrm{T}} \\
& =\eta \Omega_{(2)}^{\prime} J_{(2)}^{\prime} \Omega_{(2)}^{\prime \mathrm{T}} .
\end{aligned}
$$

On the other hand

$$
\Sigma_{2}^{\prime} \eta \Sigma_{2}^{\prime \mathrm{T}}=\eta \Omega_{(2)}^{\prime} J_{(2)}^{\prime} \Omega_{(2)}^{\prime \mathrm{T}} .
$$

Thus, determining all possible $\Sigma_{2}^{\prime}$ we will also find $\Sigma_{2}$ among them. Concluding, we can restrict our attention to the unprimed cases only. 
Finally, only the cases (2), (4), (5), and (9) remain. We determine orthogonal matrices $\Omega$ for these cases using Eq. (93). We get

$$
\begin{aligned}
& \Omega_{(2)}=\frac{1}{\sqrt{2}}\left(\begin{array}{cccc}
1 & 0 & -1 & 0 \\
0 & \sqrt{2} & 0 & 0 \\
1 & 0 & 1 & 0 \\
0 & 0 & 0 & \sqrt{2}
\end{array}\right), \quad \varepsilon_{1}=\varepsilon_{2}=-1 ; \\
& \Omega_{(4)}=\frac{1}{\sqrt{2}}\left(\begin{array}{cccc}
1 & \varepsilon_{1} & 0 & 0 \\
-\varepsilon_{1} & 1 & 0 & 0 \\
0 & 0 & \sqrt{2} & 0 \\
0 & 0 & 0 & \sqrt{2}
\end{array}\right), \quad \varepsilon_{2}=\varepsilon_{3}=-1 ; \\
& \Omega_{(5)}=I, \quad \varepsilon_{1}=1, \quad \varepsilon_{2}=\varepsilon_{3}=\varepsilon_{4}=-1 ; \\
& \Omega_{(9)}=\frac{1}{\sqrt{2}}\left(\begin{array}{cccc}
1 & 1 & 0 & 0 \\
-1 & 1 & 0 & 0 \\
0 & 0 & \sqrt{2} & 0 \\
0 & 0 & 0 & \sqrt{2}
\end{array}\right), \quad \varepsilon_{1}=\varepsilon_{2}=-1 .
\end{aligned}
$$

Now, we want to determine the corresponding $\Sigma$ matrices with the help of Eq. (96). This equation does not determine $\Sigma$ uniquely. Note that if $\Sigma$ is a solution of Eq. (96) also

$$
\Sigma^{\prime}=\Sigma \Lambda^{\mathrm{T}}
$$

where $\Lambda$ is a Lorentz transformation, is a solution. We use this freedom to determine the simplest form of $\Sigma$. Matrix $\Sigma$ corresponds to a two-qubit quantum state $\rho$ via the relation analogous to Eq. (2):

$$
\rho=\frac{1}{4} \sum_{\mu, \nu=0}^{3} \Sigma^{\mu \nu} \sigma_{\mu} \otimes \sigma_{\nu} .
$$

Therefore, even taking into account normalization, we have to assume that

$$
\Sigma^{00} \neq 0
$$

Moreover, the reduced density matrices

$$
\begin{aligned}
& \rho_{B}=\operatorname{Tr}_{A}(\rho)=\frac{1}{2} \sum_{\nu=0}^{3} \Sigma^{0 \nu} \sigma_{\nu}, \\
& \rho_{A}=\operatorname{Tr}_{B}(\rho)=\frac{1}{2} \sum_{\mu=0}^{3} \Sigma^{\mu 0} \sigma_{\mu}
\end{aligned}
$$


are positive semidefinite. It follows that

$$
\begin{aligned}
& \left(\Sigma^{00}\right)^{2}-\left(\Sigma^{01}\right)^{2}-\left(\Sigma^{02}\right)^{2}-\left(\Sigma^{03}\right)^{2} \geq 0, \\
& \left(\Sigma^{00}\right)^{2}-\left(\Sigma^{10}\right)^{2}-\left(\Sigma^{20}\right)^{2}-\left(\Sigma^{30}\right)^{2} \geq 0 .
\end{aligned}
$$

When we perform transformation defined in Eq. (108), rows of $\Sigma$ transform like four-vectors under $\Lambda$. Taking into account Eq. (113), we see that the first row of the matrix $\Sigma$ transforms under Lorentz transformations like a time-like or light-like fourvector. Therefore, using the freedom guaranteed by Eq. (108), the form of $\Sigma$ can be reduced to:

$$
\Sigma^{t}=\left(\begin{array}{cccc}
\alpha^{0} & 0 & 0 & 0 \\
\beta^{0} & \beta^{1} & 0 & 0 \\
\gamma^{0} & \gamma^{1} & \gamma^{2} & 0 \\
\delta^{0} & \delta^{1} & \delta^{2} & \delta^{3}
\end{array}\right)
$$

in a time-like case and it can be reduced to

$$
\Sigma^{l}=\left(\begin{array}{cccc}
\alpha^{0} & \varepsilon \alpha^{0} & 0 & 0 \\
\beta^{0} & \beta^{1} & \beta^{2} & 0 \\
\gamma^{0} & \gamma^{1} & \gamma^{2} & \gamma^{3} \\
\delta^{0} & \delta^{1} & \delta^{2} & \delta^{3}
\end{array}\right), \quad \varepsilon= \pm 1
$$

in a light-like case. In both cases, (115) and (116), it holds [compare Eq. (114)]

$$
0 \neq\left(\alpha^{0}\right)^{2} \geq\left(\beta^{0}\right)^{2}+\left(\gamma^{0}\right)^{2}+\left(\delta^{0}\right)^{2}
$$

Now, we insert (115) and (116) into Eq. (96) for $\Omega$ and $J$ given in Eqs. $(78,104)$ or in Eqs. $(81,105)$ or in Eqs. $(84,106)$ or in Eqs. $(89,107)$. Solving obtained conditions we arrive at the following possibilities:

Case number (2), time-like first row:

$$
\begin{gathered}
\Sigma_{(2)}^{t}=\left(\begin{array}{cccc}
\sqrt{\lambda_{1}} & 0 & 0 & 0 \\
\frac{1}{\sqrt{2 \lambda_{1}}} & \varepsilon \frac{\sqrt{2 \lambda_{1}^{2}+1}}{\sqrt{2 \lambda_{1}}} & 0 & 0 \\
0 & \varepsilon \frac{\sqrt{\lambda_{1}}}{\sqrt{2 \lambda_{1}^{2}+1}} & \varepsilon^{\prime} \lambda_{1} \frac{\sqrt{2 \lambda_{1}}}{\sqrt{2 \lambda_{1}^{2}+1}} & 0 \\
0 & 0 & 0 & \varepsilon^{\prime \prime} \sqrt{\lambda_{2}}
\end{array}\right), \\
\lambda_{1} \geq \frac{1}{\sqrt{2}}, \quad \varepsilon= \pm 1, \quad \varepsilon^{\prime}= \pm 1, \quad \varepsilon^{\prime \prime}= \pm 1
\end{gathered}
$$

Case number (2), light-like first row: this case is contradictory. 
Case number (4), time-like first row:

$$
\begin{gathered}
\Sigma_{(4)}^{t}=\left(\begin{array}{cccc}
\sqrt{\lambda_{1}+\frac{\varepsilon_{1}}{2}} & 0 & 0 & 0 \\
\frac{1}{2 \sqrt{\lambda_{1}+\frac{\varepsilon_{1}}{2}}} & \varepsilon \frac{\lambda_{1}}{\sqrt{\lambda_{1}+\frac{\varepsilon_{1}}{2}}} & 0 & 0 \\
0 & 0 & \varepsilon^{\prime} \sqrt{\lambda_{2}} & 0 \\
0 & 0 & 0 & \varepsilon^{\prime \prime} \sqrt{\lambda_{3}}
\end{array}\right), \\
\lambda_{1} \geq \frac{1-\varepsilon_{1}}{\sqrt{2}}, \quad \varepsilon_{1}= \pm 1, \quad \varepsilon= \pm 1, \quad \varepsilon^{\prime}= \pm 1, \quad \varepsilon^{\prime \prime}= \pm 1 ;
\end{gathered}
$$

Case number (4), light-like first row:

$$
\Sigma_{4}^{l}=\left(\begin{array}{cccc}
\alpha^{0} & -\alpha^{0} & 0 & 0 \\
\beta^{0} & \beta^{1} & \beta^{2} & 0 \\
\gamma^{0} & -\gamma^{0} & \gamma^{2} & \gamma^{3} \\
\delta^{0} & -\delta^{0} & \delta^{2} & \delta^{3}
\end{array}\right)
$$

with the condition (117) and

$$
\begin{array}{ll}
\lambda_{1}=\frac{1}{2}, & 1+\left(\beta^{0}\right)^{2}=\left(\beta^{1}\right)^{2}+\left(\beta^{2}\right)^{2}, \\
\frac{1}{2 \alpha^{0}}=\beta^{0}+\beta^{1}, & \gamma^{2} \delta^{2}+\gamma^{3} \delta^{3}=0, \\
\lambda_{2}=\left(\gamma^{2}\right)^{2}+\left(\gamma^{3}\right)^{2}, & \lambda_{3}=\left(\delta^{2}\right)^{2}+\left(\delta^{3}\right)^{2}, \\
\gamma^{0} \delta^{2}=\delta^{0} \gamma^{2} . &
\end{array}
$$

Case number (5), time-like first vector:

$$
\begin{aligned}
\Sigma_{5}^{t} & =\left(\begin{array}{cccc}
\sqrt{\lambda_{1}} & 0 & 0 & 0 \\
0 & \varepsilon \sqrt{\lambda_{2}} & 0 & 0 \\
0 & 0 & \varepsilon^{\prime} \sqrt{\lambda_{3}} & 0 \\
0 & 0 & 0 & \varepsilon^{\prime \prime} \sqrt{\lambda_{4}}
\end{array}\right), \\
\varepsilon & = \pm 1, \quad \varepsilon^{\prime}= \pm 1, \quad \varepsilon^{\prime \prime}= \pm 1
\end{aligned}
$$

Case number (5), light-like first row:

$$
\Sigma_{5}^{l}=\left(\begin{array}{cccc}
\alpha^{0} & \varepsilon \alpha^{0} & 0 & 0 \\
\beta^{0} & \varepsilon \beta^{0} & \beta^{2} & 0 \\
\gamma^{0} & \varepsilon \gamma^{0} & \gamma^{2} & \gamma^{3} \\
\delta^{0} & \varepsilon \delta^{0} & \delta^{2} & \delta^{3}
\end{array}\right)
$$

with the conditions (117), $\lambda_{1}=0$, and we have three possibilities in this case:

$$
\begin{array}{ll}
\beta^{2}=0 & \gamma^{2} \delta^{2}+\gamma^{3} \delta^{3}=0 \\
\lambda_{3}=\left(\gamma^{2}\right)^{2}+\left(\gamma^{3}\right)^{2}, & \lambda_{4}=\left(\delta^{2}\right)^{2}+\left(\delta^{3}\right)^{2},
\end{array}
$$


or

$$
\gamma^{2}=0, \quad \delta^{2}=0, \quad \gamma^{3}=0, \quad\left(\delta^{3}\right)^{2}=\lambda_{4},
$$

or

$$
\gamma^{2}=0, \quad \delta^{2}=0, \quad\left(\gamma^{3}\right)^{2}=\lambda_{3}, \quad \delta^{3}=0 .
$$

Case number (9), time-like first row:

$$
\begin{gathered}
\Sigma_{9}^{t}=\left(\begin{array}{cccc}
\sqrt{\sigma} & 0 & 0 & 0 \\
\frac{\tau}{\sqrt{\sigma}} & \varepsilon \frac{\sqrt{\sigma^{2}+\tau^{2}}}{\sqrt{\sigma}} & 0 & 0 \\
0 & 0 & \varepsilon^{\prime} \sqrt{\lambda_{1}} & 0 \\
0 & 0 & 0 & \varepsilon^{\prime \prime} \sqrt{\lambda_{2}}
\end{array}\right), \\
\sigma^{2} \geq \tau^{2}, \quad \varepsilon= \pm 1, \varepsilon^{\prime}= \pm 1, \varepsilon^{\prime \prime}= \pm 1 ;
\end{gathered}
$$

Case number (9), light-like first row:

$$
\Sigma_{9}^{l}=\left(\begin{array}{cccc}
\alpha^{0} & \varepsilon \alpha^{0} & 0 & 0 \\
\beta^{0} & \beta^{1} & \beta^{2} & 0 \\
\gamma^{0} & \varepsilon \gamma^{0} & \gamma^{2} & \gamma^{3} \\
\delta^{0} & \varepsilon \delta^{0} & \delta^{2} & \delta^{3}
\end{array}\right)
$$

with the conditions (117) and

$$
\begin{aligned}
\sigma & =0, & \tau \neq 0 & \\
\varepsilon & = \pm 1, & & \left(\beta^{0}\right)^{2}=\left(\beta^{1}\right)^{2}+\left(\beta^{2}\right)^{2}, \\
\tau & =\alpha^{0}\left(\beta^{0}-\varepsilon \beta^{1}\right), & & \gamma^{2} \delta^{2}+\gamma^{3} \delta^{3}=0 \\
\beta^{2} \gamma^{2} & =\gamma^{0}\left(\beta^{0}-\varepsilon \beta^{1}\right), & & \beta^{2} \delta^{2}=\delta^{0}\left(\beta^{0}-\varepsilon \beta^{1}\right), \\
\lambda_{1} & =\left(\gamma^{2}\right)^{2}+\left(\gamma^{3}\right)^{2}, & & \lambda_{2}=\left(\delta^{2}\right)^{2}+\left(\delta^{3}\right)^{2} .
\end{aligned}
$$

Now, all of the matrices $(118,120,122,127,129,134,136)$ correspond to two-qubit quantum states via the relation (109). Therefore, after the transformation

$$
R=\Lambda_{1} \Sigma \Lambda_{2}^{T}
$$

the state (109) changes according to Eq. (4). Thus, the matrix $R$ from Eq. (142) has to fulfill conditions analogous to Eqs. $(113,114)$. To proceed further, we take $\Lambda_{2}=I$ and $\Lambda_{1}=B(\mathbf{n}, \xi)$ (boost in the direction of the vector $\mathbf{n}$ with rapidity $\xi$ ) and calculate the matrices $B(\hat{\mathbf{x}}, \xi) \Sigma, B(\hat{\mathbf{z}}, \xi) \Sigma$. Next, the first rows of the matrices $B(\hat{\mathbf{x}}, \xi) \Sigma, B(\hat{\mathbf{z}}, \xi) \Sigma$ should fulfill the condition analogous to (113) for all values of $\xi$. Using this method, we can show that the matrices $\Sigma_{2}^{t}, \Sigma_{4}^{l}, \Sigma_{9}^{t}, \Sigma_{9}^{l}$ cannot correspond to quantum states. Moreover, we can restrict the domain of parameters in the cases $\Sigma_{4}^{t}$ and $\Sigma_{5}^{l}$. We can show with the help of this method that in the case of $\Sigma_{4}^{t}$ [Eq. (120)], we have to put 
$\varepsilon_{1}=1$ (which implies that in this case $\lambda_{1} \geq 0$ ) and in the case of $\Sigma_{5}^{l}$ [Eq. (129)] we have to put $\beta^{2}=\gamma^{2}=\gamma^{3}=\delta^{2}=\delta^{3}=0$.

Let us remind here that our aim is to find the simplest, inequivalent $\Sigma$ s from which all possible $R$ s corresponding to quantum states can be generated via Eq. (142). We can use this observation to further simplify the remaining cases $\Sigma_{4}^{t}, \Sigma_{5}^{t}, \Sigma_{5}^{l}$.

First of all, in $\Sigma_{5}^{l}$ the first two columns are proportional and the last two columns consist of zeros. Therefore, with the help of appropriate Lorentz transformation acting on the left-hand side we can transform $\Sigma_{5}^{l}$ to one of the following forms (depending on the character of the first column):

$$
\Sigma_{5}^{l l}=\left(\begin{array}{cccc}
\alpha^{0} & \varepsilon \alpha^{0} & 0 & 0 \\
0 & 0 & 0 & 0 \\
0 & 0 & 0 & 0 \\
0 & 0 & 0 & 0
\end{array}\right), \quad \varepsilon= \pm 1
$$

or

$$
\Sigma_{5}^{l t}=\left(\begin{array}{cccc}
\alpha^{0} & \varepsilon \alpha^{0} & 0 & 0 \\
\varepsilon \alpha^{0} & \alpha^{0} & 0 & 0 \\
0 & 0 & 0 & 0 \\
0 & 0 & 0 & 0
\end{array}\right), \quad \varepsilon= \pm 1
$$

Next, taking

$$
\Lambda_{1}=\Lambda_{2}=\left(\begin{array}{cccc}
1 & 0 & 0 & 0 \\
0 & -1 & 0 & 0 \\
0 & 0 & -1 & 0 \\
0 & 0 & 0 & 1
\end{array}\right)
$$

we can transform $\Sigma_{5}^{l l}, \Sigma_{5}^{l t}$ with $\varepsilon=-1$ to $\Sigma_{5}^{l l}, \Sigma_{5}^{l t}$ with $\varepsilon=1$. Thus, from the case (5) with the light-like first row [Eq. (129)], we obtain two inequivalent possibilities

$$
\Sigma_{5}^{l l}=\alpha^{0}\left(\begin{array}{cccc}
1 & 1 & 0 & 0 \\
0 & 0 & 0 & 0 \\
0 & 0 & 0 & 0 \\
0 & 0 & 0 & 0
\end{array}\right), \quad \Sigma_{5}^{l t}=\alpha^{0}\left(\begin{array}{cccc}
1 & 1 & 0 & 0 \\
1 & 1 & 0 & 0 \\
0 & 0 & 0 & 0 \\
0 & 0 & 0 & 0
\end{array}\right)
$$

$\Sigma_{4}^{t}$ can be re-parametrized in the following form:

$$
\Sigma_{4}^{t}=a\left(\begin{array}{cccc}
1 & 0 & 0 & 0 \\
y^{\prime} & \varepsilon\left(1-y^{\prime}\right) & 0 & 0 \\
0 & 0 & x^{\prime} & 0 \\
0 & 0 & 0 & z^{\prime}
\end{array}\right)
$$

where $y^{\prime}=\frac{1}{2 \lambda_{1}+1} \in(0,1\rangle, a=\sqrt{\lambda_{1}+\frac{1}{2}} \geq \frac{1}{\sqrt{2}}, x^{\prime}=\varepsilon^{\prime} \sqrt{\frac{\lambda_{2}}{\lambda_{1}+1 / 2}} \in \mathbb{R}, z^{\prime}$ $=\varepsilon^{\prime \prime} \sqrt{\frac{\lambda_{3}}{\lambda_{1}+1 / 2}} \in \mathbb{R}$. 
$\Sigma_{5}^{t}$ can be rewritten as

$$
\Sigma_{5}^{t}=b\left(\begin{array}{cccc}
1 & 0 & 0 & 0 \\
0 & X & 0 & 0 \\
0 & 0 & Y & 0 \\
0 & 0 & 0 & Z
\end{array}\right)
$$

where $b=\sqrt{\lambda_{1}}, X=\varepsilon \sqrt{\frac{\lambda_{2}}{\lambda_{1}}}, Y=\varepsilon^{\prime} \sqrt{\frac{\lambda_{3}}{\lambda_{1}}}, Z=\varepsilon^{\prime \prime} \sqrt{\frac{\lambda_{4}}{\lambda_{1}}}$.

Now, let us notice that matrices $\Sigma$ and $\alpha \Sigma(\alpha \in \mathbb{R}, \alpha \neq 0)$ correspond to the same density matrix $\rho$ since we normalize $\rho$ to unit trace. Therefore, we can omit the overall factors in Eqs. $(146,147,148)$. Next, $\Sigma$ s from Eqs. $(146,147,148)$ correspond to positive semidefinite matrices via the relation (109) iff all of the coefficients (3) are nonnegative. tive.

For $\Sigma_{5}^{l l}$ and $\Sigma_{5}^{l t}$ from Eq. (146), all of the coefficients (3) are identically nonnega-

For $\Sigma_{4}^{t}$ from Eq. (147), nonnegativity of the coefficients (3) implies:

$$
\begin{array}{ll}
x^{\prime}=z^{\prime} & \text { for } \varepsilon=-1, \\
x^{\prime}=-z^{\prime} & \text { for } \varepsilon=1,
\end{array}
$$

and

$$
x^{\prime 2} \leq 1-y^{\prime}
$$

Moreover, the cases with $\varepsilon=1$ and $\varepsilon=-1$ [Eqs. $(149,150)]$ are connected via the relation

$$
\left.\Sigma_{4}^{t}\right|_{\varepsilon=1} \Lambda_{2}^{T}=\left.\Sigma_{4}^{t}\right|_{\varepsilon=-1},
$$

where $\Lambda_{2}$ is given in Eq. (145). Therefore, the case 4 with the time-like first row [Eq. (120)] reduces to (147) with $\varepsilon=-1, z^{\prime}=x^{\prime}$, and with the condition given in Eq. (151). Therefore,

$$
\Sigma_{4}^{t}=\left(\begin{array}{cccc}
1 & 0 & 0 & 0 \\
0 & x^{\prime} & 0 & 0 \\
0 & 0 & x^{\prime} & 0 \\
y^{\prime} & 0 & 0 & y^{\prime}-1
\end{array}\right)
$$

with $x^{\prime} \in\langle-1,1\rangle, 0<y^{\prime} \leq 1-x^{\prime 2}$, (note that (153) can be obtained from (147) with the conditions $(149,150,152)$ via the appropriate permutation).

Now, for $y^{\prime} \neq 1$ we can symmetrize (153) via Lorentz boosts. That is why we exclude the case $y^{\prime} \neq 1$ and treat it separately [compare (8)]. The symmetrized form of (153) is given in Eq. (12). Note that

$$
x=\frac{4 x^{\prime}}{y^{\prime}+2 \sqrt{1-y^{\prime}}}, \quad y=\frac{y^{\prime}}{y^{\prime}+2 \sqrt{1-y^{\prime}}} .
$$

For $\Sigma_{5}^{t}$ from Eq. (148), nonnegativity of the coefficients (3) implies the conditions given in Eq. (11). Moreover, applying transformations from $L_{+}^{\uparrow}$, we can rearrange $X$, $Y, Z$ in such a way that $1 \geq X \geq Y \geq|Z|$. 
This completes the proof of the Theorem 1 .

\section{References}

1. Horodecki, R., Horodecki, P., Horodecki, M., Horodecki, K.: Quantum entanglement. Rev. Mod. Phys. 81, 865 (2009)

2. Modi, K., Brodutch, A., Cable, H., Paterek, T., Vedral, V.: The classical-quantum boundary for correlations: discord and related measures. Rev. Mod. Phys. 84, 1655 (2012)

3. Caban, P., Podlaski, K., Rembieliński, J., Smoliński, K.A., Walczak, Z.: Entanglement and tensor product decomposition for two fermions. J. Phys. A Math. Gen. 38, L79 (2005)

4. Verstraete, F., Dehaene, J., DeMoor, B.: Local filtering operations on two qubits. Phys. Rev. A 64, 010101(R) (2001)

5. Bengtsson, I., Życzkowski, K.: Geometry of Quantum States. Cambridge University Press, Cambridge (2006)

6. Wootters, W.K.: Entanglement of formation of an arbitrary state of two qubits. Phys. Rev. Lett. 80, 2245 (1998)

7. Olivier, H., Zurek, W.H.: Quantum discord: a measure of the quantumness of correlations. Phys. Rev. Lett. 88, 017901 (2002)

8. Luo, S., Fu, S.: Geometric measure of quantum discord. Phys. Rev. A 82, 034302 (2010)

9. Okrasa, M., Walczak, Z.: Quantum discord and multipartite correlations. EPL 96, 60003 (2011)

10. Luo, S.: Quantum discord for two-qubit systems. Phys. Rev. A 77, 042303 (2008)

11. Ali, M., Rau, A.R.P., Alber, G.: Quantum discord for two-qubit X states. Phys. Rev. A 81, 042105 (2010)

12. Huang, Y.: Quantum discord for two-qubit X states: analytical formula with very small worst-case error. Phys. Rev. A 88, 014302 (2013)

13. Giorda, P., Paris, M.G.A.: Gaussian quantum discord. Phys. Rev. Lett. 105, 020503 (2010)

14. Adesso, G., Datta, A.: Quantum versus classical correlations in Gaussian states. Phys. Rev. Lett. 105, 030501 (2010)

15. Li, B., Wang, Z.X., Fei, S.M.: Quantum discord and geometry for a class of two-qubit states. Phys. Rev. A 83, 022321 (2011)

16. Chitambar, E.: Quantum correlations in high-dimensional states of high symmetry. Phys. Rev. A 86, 032110 (2012)

17. Huang, Y.: Computing quantum discord is NP-complete. New J. Phys. 16, 033027 (2014)

18. Girolami, D., Adesso, G.: Quantum discord for general two-qubit states: analytical progress. Phys. Rev. A 83, 052108 (2011)

19. Gohberg, I., Lancaster, P., Rodman, L.: Indefinite Linear Algebra and Applications. Birkhäuser, BaselBoston-Berlin (2005) 